\section{Urs Lütolf}

Wir sitzen in der Nordstube des Torkelhauses in der Untergasse von Sargans. Das von den mütterlichen Vorfahren bewohnte Gebäude wurde nach dem grossen Brand von 1811 wieder aufgebaut. In der Dämmerung sind gerade noch die kleinen Buchsbaumbüsche und kahlen Rebstöcke auf der Südseite bemerkbar.

\section{Herr Lütolf, was war früher?}

Ich bin 1945 geboren und in Winterthur aufgewachsen. Die ersten zwei Lebensjahre verbrachte ich im Burghölzli, wo mein Vater als Psychiatrieassistent arbeitete. Damals lebten auch alle Ärzte auf dem Areal und so bin ich oft von Patienten gehütet worden, oder mein Bett wurde gelegentlich nachts in den Hörsaal gestellt, wenn ich den Schlaf meiner Eltern störte. Ein Chemie- oder Physikstudium hätte mich auch interessiert, ich habe mich dann aber doch für die Medizin entschieden. Vielleicht weil ich in einer Allgemeinpraxis aufgewachsen bin, denn meine Mutter war Allgemeinärztin und mein Vater hatte als Psychiater 1947 in Winterthur noch nicht genügend Patienten für eine Existenz als Facharzt. Ich habe nach den Vorprüfungen in Genf 1971 das Staatsexamen in Zürich abgelegt. Als völlig unpolitischer Bürger erlebte ich zufällig die Maiunruhen und den Generalstreik von 1968 in Paris. Ausser Strassenkämpfen gab es die engagierten Diskussionen auf allen Stockwerken der "Ecole de Médecine», was im "Le Livre Blanc des Réformes" resultierte. Das war mein erster Kontakt mit Studienreformen. Nach einem Jahr Chirurgie in Wattwil und drei Jahren Nuklearmedizin und Strahlentherapie am Universitätsspital habe ich in San Francisco kardiologische Funktionsprüfungen entwickelt. In Zürich konnten wir so mit unseren nuklearmedizinischen Funktionsprüfungen zum Gelingen der ersten Koronardilatationen beitragen. Ich wollte aber wieder direkt mit Patienten zu tun haben, verlagerte meine medizinischen Interessen auf die Radioonkologie und war von 1982 bis 1988 Chefarzt der Radioonkologie am Kantonsspital St. Gallen. 1988 wurde ich ordentlicher Professor am Universitätsspital Zürich, wo ich seither die radioonkologische Klinik leite. Von 1990 bis 2000 war ich Studiendekan, und seit 1996 leite ich zusätzlich das Departement für Medizinische Radiologie.

Können Sie uns sagen, wie sich Ihre Arbeit aufteilt? Dazu gehört die klinische Arbeit mit Abteilungsvisiten, Rapporten und einer Sprechstunde. Neben der Bestrahlungsplanung und Simulation für Patienten leite ich täglich um 16 Uhr die gemeinsame Vorstellung aller Patienten, etwa 1400 pro Jahr. Dazu kommen verschiedene Konsilien und interdisziplinäre Sprechstunden. Weiter beschäftigt mich die klinische Forschung, u.a. die Entwicklung neuer Technologien, zum Teil in Zusammenarbeit mit der ETH, weiter pro-

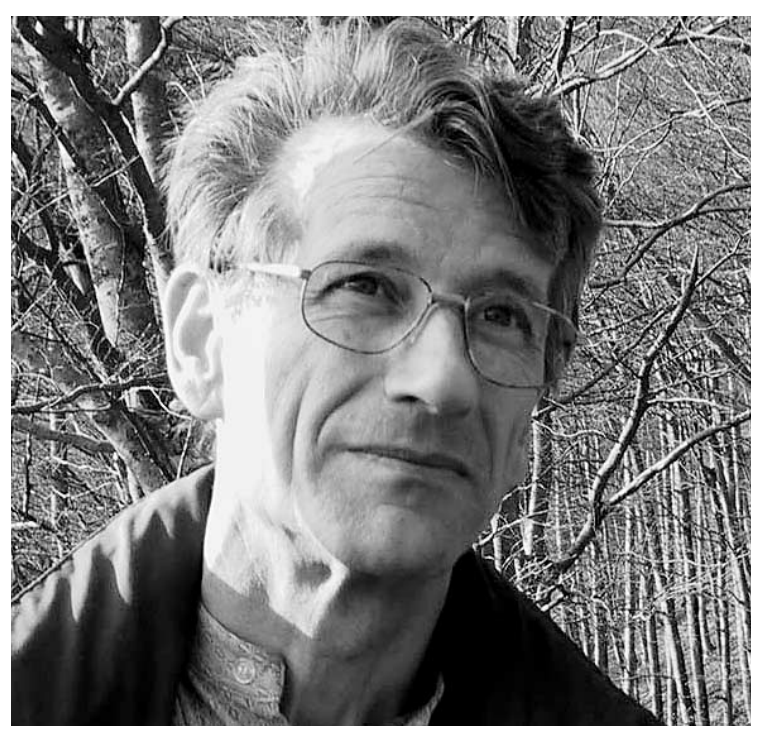

spektive Vergleichsstudien zur Chemo- und Radiotherapie bei ORL- und andern Tumoren und molekularbiologische Untersuchungen der Signalübertragungen von Zellen unter Bestrahlung, an denen ich nur indirekt durch Bereitstellung der Infrastruktur beteiligt bin. Drittens bin ich als Lehrer tätig, halte Vorlesungen und leite selber ein Tutorat mit Kleingruppen von 6 bis 8 Studenten im 6. Jahr. Diese aufwendige Unterrichtsform muss für 240 Studierende für je ein Winter- und Sommersemester organisiert werden. Wir führen unter Leitung eines Dozenten interdisziplinär ein «wildes» Tutorat im 4. Studienjahr für Freiwillige in «molekularer Onkologie» während eines Semesters. Das ist Elite- und Nachwuchsförderung für besonders motivierte Studierende. Dazu kommen die Absolventen des Wahlstudienjahrs oder ein Poliklinik-Morgen, an dem bis zu 30 Teilnehmer unter Aufsicht der Assistenten und Oberärzte Patienten untersuchen und Therapiepläne besprechen können. Eine Idee aus der Zeit als Studiendekan ist es, den Studierenden in verschiedenen Fächern eine "Heimatklinik» anzubieten. Dabei sollen die Hälfte oder ein Drittel durch intensive Kontakte wie Visiten, Fragestunden und Internetbenützung in der Anonymität des grossen Betriebes eine gewisse Geborgenheit bei einer Klinik finden. $\mathrm{Zu}$ erwähnen sind noch die überregionale Assistentenausbildung, die Dissertanten und die Fortbildungstätigkeit an Kongressen und Vorträgen.

Was hat sich im Umfeld des USZ geändert? In der Schweiz gibt es zurzeit 15 Zentren für Radioonkologie, die eine Basistherapie anbieten. Wir haben Verträge mit Chur und Münsterlingen und stellen Vertretungen sicher. Mich interessiert speziell die Planung und Konzeptentwicklung, damit möglichst ökonomisch sinnvolle Lösungen erreicht werden. Das geschieht auf eigene Initiative und ist nicht immer willkommen. Was die Konzepte durchkreuzen kann, ist die Eröffnung von Strahlentherapien in privaten Kliniken. Damit ist oft die Abwanderung von Fachleuten verbunden. Für die öffentlichen Spitäler gehen Kenntnisse verloren, und die Steuerzahler kostet es 
unter Umständen mehr, weil oft finanzielle Überlegungen die medizinischen Indikationen beeinflussen. Zum Beispiel, wenn bei Rückenmetastasen 20 Sitzungen statt 4 Sitzungen, trotz gleichem Resultat, wegen mehr Einnahmen angeordnet werden. Da wären Pauschalen vernünftig, doch die Kassen sind daran gar nicht besonders interessiert. Weil es imagemässig gut schien, wurde von den Kassen freiwillig ein Tarif fürs Gamma-Knife zur Therapie von Hirntumoren und Hirnmetastasen gewährt, der ein Mehrfaches einer ebenbürtigen Linearbeschleunigertherapie beträgt. Aber auch intern sind Tarifgestaltung oft betriebswirtschaftlich nicht nachzuvollziehen; hier muss noch viel geschehen.

\section{Was halten Sie von vermehrtem Sponsoring?}

Eine Zusammenarbeit mit Privaten ist notwendig. So haben zum Beispiel die Krebsliga und ein Hersteller von technischer Ausrüstung eine Physikerstelle für ein Jahr bezahlt, damit wir die neuen Bestrahlungstechniken einführen können. Für die Pharmaindustrie sind wir nicht sehr interessant. Auch dafür ein Beispiel: Das Zungengrundkarzinom kann mit einer neuen Bestrahlungstechnik so behandelt werden, dass die Speicheldrüsen in $80 \%$ geschont werden. Eine zusätzliche Physikerstelle wäre Voraussetzung, diese Therapie für alle Patienten verfügbar zu machen. Die teurere Behandlung mit einem strahlenschützenden Medikament, das nur in 40\% erfolgreich ist, wird ohne weiteres bezahlt. Das neue Bestrahlungsverfahren ist wirksamer und auf alle Patienten bezogen billiger, doch der vorgegebene Stellenplan lässt diese vernünftigere Alternative nicht zu.

Was können wir von der Bologna-Reform erwarten? Der vorgesehene Zwischenabschluss (Bachelor nach dem 3. Propädeutikum) ist weder Fisch noch Vogel. Wer Medizin studiert, wird nur mit klinischer Tätigkeit vom Studium Gebrauch machen können. Die Bologna-Reform ist für die Medizin auch nirgends in Europa ein Thema. Ich habe in der Fleiner-II-Kommission (Ausbildungsgesetz) eine spannende Zeit erlebt. Die Arbeit der ersten Kommission (Weiterbildung) musste ja wegen den bilateralen Verträgen zuerst abgeschlossen werden. Jetzt sind die Resultate beider bundesrätlichen Kommissionen als "Medizinalberufsgesetz» durch die Vernehmlassung und kommen wohl im Frühjahr 2002 ins Parlament. Den sechs Jahren Mittelschule werden sechs Jahre Studium folgen mit etwa 80\% vorgegebenen Kernfächern (Kernstudium) und 20\% Wahlfächern (Mantelstudium). Nach letzterem werden die Fakultäten ihre Angebote individuell gestalten können; Zürich ist jetzt daran, die Studienrichtungen auszudividieren. Für den ganzen Hochschulbereich wird die Akkreditierung eingeführt, so auch für die Medizinerausbildung unter der Leitung von Herrn Heusser (BBW, Leitung Herr Klaiber im Departement Dreifuss).

Und die Weiterbildung?

Diese wird im Zuge der Freizügigkeit des Personenverkehrs (bilaterale Verträge mit der EU) bekanntlich vom Bund übernommen und zur Ausführung zurück an die FMH delegiert. Allerdings ist jetzt der Staat für die Kontrolle verantwortlich. Frau Dreifuss hat einen Weiterbildungsausschuss bestimmt, den Herr Salzberg präsidiert, während ich Vize bin. Es wird eine Gratwanderung zwischen Reglementierung und akademischer Freiheit werden.

Warum sind unsere Universitäten bei der freiwilligen Akkreditierung vor 3 Jahren nicht besonders gut weggekommen?

Eine Gruppe von Experten aus den USA, Holland, England und Deutschland hat die Strukturen unserer Fakultäten untersucht. Diese Leute waren auch bei uns während drei Tagen auf Besuch. Ich musste als Studiendekan eine Selbstanalyse der strukturellen Stärken und Schwächen ausarbeiten, war dann aber bei der «Übungsbesprechung» von der Fakultät sehr alleingelassen. Damals beschloss ich übrigens, meine Verpflichtung als Studiendekan nach 10 Jahren zu beenden. Mit einer neu strukturierten Kommission "Lehre» meine ich dafür gesorgt zu haben, dass sich die Reform auf breiterer Basis entwickeln kann. Ich glaube, dass wir relativ wenig Verständnis für unsere Ausbildung fanden, weil die Akkreditierung und Kontrolle in den USA aus historischen Gründen viel wichtiger sind. Dort sind seit langem private und öffentliche Medical Schools nebeneinander, ein Organ müsste Einfluss und Kontrolle haben. Für die Experten war es zum Beispiel unverständlich, dass wir die Studierenden nach dem 3. Propädeutikum bis zum Staatsexamen nicht mehr prüfen.

Ein erster "Ernstfall» der Akkreditierung dürfte die geplante Fakultät der Hirslanden werden, die das Klinikum, nach dem Propädeutikum in Freiburg, in den eigenen Privatspitälern plant. Von mir aus ist Konkurrenz willkommen, das hat auch positive Wirkungen.

Wie wird es für den Nachwuchs in 10 Jahren sein? Ich bin nicht sicher, ob die Möglichkeiten der Mobilität für Studierende in der Schweiz und in Europa besser werden, weil Verschulung und Quantifizierung massiv zunehmen. Die Zukunft wird enger. Die Tendenz zur Fachhochschule ist klar. Zum Teil muss die Selektion vorgenommen werden, die in der Mittelschule nicht mehr stattfindet. Das Profil des fleissigen, überdurchschnittlich angepassten Studierenden gab es in der Medizin seit meinem Studium, aber ein Mentalitätswechsel ist absehbar. Ein neuer Typ Mediziner wächst heran, für den die Arbeitszeitbeschränkung die konsequente Ausrichtung auf die zu erwartenden Berufsaussichten ist. Daneben dürfen wir die hochmotivierten Studierenden aber nicht verlieren, die für unsere wissenschaftlichen Fortschritte und Topmedizin Motoren sein werden.

Eine Mitteilung an unsere Leserschaft?

Ja - Technik und Humanität lassen sich durchaus kombinieren. Sie sind nicht ein Gegensatz per se.

Interview: Dr. med. Erhard Taverna 